\title{
Acoustic parametric techniques for neutrino telescopes
}

\author{
Miguel Ardid ${ }^{1,2}$, Dídac D.Tortosa ${ }^{1,2, \star}$, Carlos David Llorens Alvarez ${ }^{1}$, Juan A. Martínez-Mora ${ }^{1,2}$, \\ and María Saldaña ${ }^{1,2}$ \\ ${ }^{1}$ Universitat Politècnica de València (UPV) \\ ${ }^{2}$ Institut d'Investigació per a la Gestió Integrada de les Zones Costaneres (IGIC). \\ cl Paranimf 1, 46730 Gandia, València, Spain
}

\begin{abstract}
In this work, we present a compact transmitter array based on the parametric acoustic sources effect able to reproduce the acoustic signature of an Ultra-High Energy neutrino interaction in water. We also propose to use directive transducers employing the parametric technique for the characterization of piezo-ceramic sensors contained in the KM3NeT Digital Optical Modules. This technique can minimize the need for tests in an anechoic tank.
\end{abstract}

\section{Introduction}

This paper describes two applications of the parametric effect useful for neutrino telescopes. The first one is related to the development of a compact array [1,2] able to reproduce the Ultra-High Energy (UHE) neutrino's acoustic signature underwater [3]. The second one is for the characterization of the acoustic sensors on a tank of moderate dimensions.

\section{Generation of the acoustic signature of a UHE neutrino with a parametric array}

The UHE neutrino's acoustic signature is very peculiar because it is very directive $\left(\sim 1^{\circ}\right.$ at $1 \mathrm{~km}$ distance) although it is composed by relatively low frequencies, between 2 to $50 \mathrm{kHz}[3]$. So, to mimic this signature is not trivial. There are two ways of trying to reproduce this signature with acoustic emitters. One possibility is to use a long linear array, such as the attempt of using 8 elements in a total length of $8 \mathrm{~m}$ to generate a bipolar acoustic pulse at about $23 \mathrm{kHz}$. The second one is by means of a compact array using parametric acoustic sources effect. Roughly speaking, this technique employs the emission of two intense waves of nearly the same high frequency (1st beam) creating combinations of these two frequencies (secondary beams) in water or ice. Specifically, the parametric effect is used to get low frequency beams (the frequency difference, 2nd beam) with a pronounced directivity [2]. So far, none of the attempts have been fully successful due to difficulties of operation for the long arrays and for the low efficiency

^e-mail: didieit@upv.es 
achieved in the compact arrays. To address the latter point, a new compact calibrator is being developed using as unit element a sensor composed by the piezo-ceramic UCE-534541, and Polyurethane EL242F and aluminum as matching and backing materials, respectively [4].

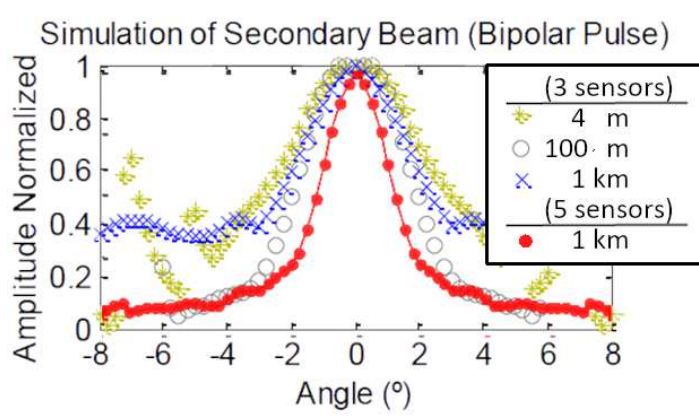

Figure 1. Experimental data (4 meters) and simulations to array.
Once the single element has been efficiently tested for this application in terms of parametric emission, waveform shape and directivity [4], the vdevelopment of the array has been initiated, both in simulations and experimentally. For instance, in Fig.1 the directivity results obtained of a first prototype with a array of $85 \mathrm{~cm}$ length with 3 elements measured at $4 \mathrm{~m}$ are presented and compared to the predictions at $1 \mathrm{~km}$ distance from simulations for a 5-element array with $14 \mathrm{~cm}$ optimum distance between elements, indicating that an opening of about $1^{\circ}$ is feasible. Simulations of an array with 5 elements reduced a directivity of $3^{\circ}$ Full Width Half Maximum.

\section{Calibration of the $\mathrm{KM} 3 \mathrm{NeT}$ acoustic sensors using the parametric technique}

Each Digital Optical Module (DOM) of KM3NeT has a piezo-ceramic sensor installed in the bottom part. To calibrate this acoustic sensor properly, an anechoic pool is usually needed [5]. Here, we present an alternative to the use of this expensive installations by using moderate size pools with parametric emitters. To test this, an experiment in a small tank of water $\left(110 \times 85 \times 80 \mathrm{~cm}^{3}\right)$ has been conducted using a commercial transducer, RESON TC3027 of $1 \mathrm{MHz}$ resonance frequency. The receiver was set to $50 \mathrm{~cm}$, i.e., in the far field (Rayleigh distance was $47 \mathrm{~cm}$ ). The signal used was a 10 to $50 \mathrm{kHz}$ sine sweep modulated with a tone of $1 \mathrm{MHz}$ with $1 \mathrm{~ms}$ duration, so to produce a frequency difference from 20 to $100 \mathrm{kHz}$ parametrically. The spectrogram of the received signal is shown in Fig. 2, where both beams can be observed, as well as the reflections in the tank. The properties of the parametric signal have also been studied and are be summarized in Fig. (??).

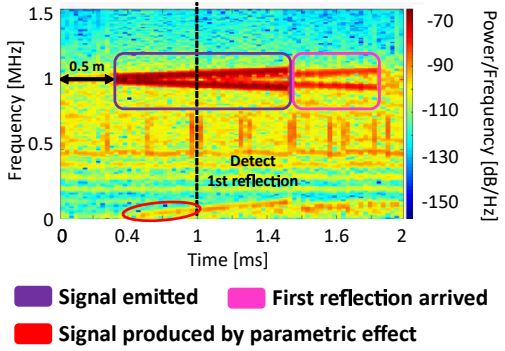

Figure 2. Spectrogram of a measure in the tank.
The problem of using a small tank is that the first reflection can overlay the direct signal if its time duration is large, but according to simulations using a small pool (e.g. $3 \times 4 \times 2$ $\mathrm{m}^{3}$ ) the first reflection with this parametric emitter appears $1.5 \mathrm{~ms}$ later than the direct signal, hence allowing for an easy discrimination in the time domain and, thus, for a clean calibration. 

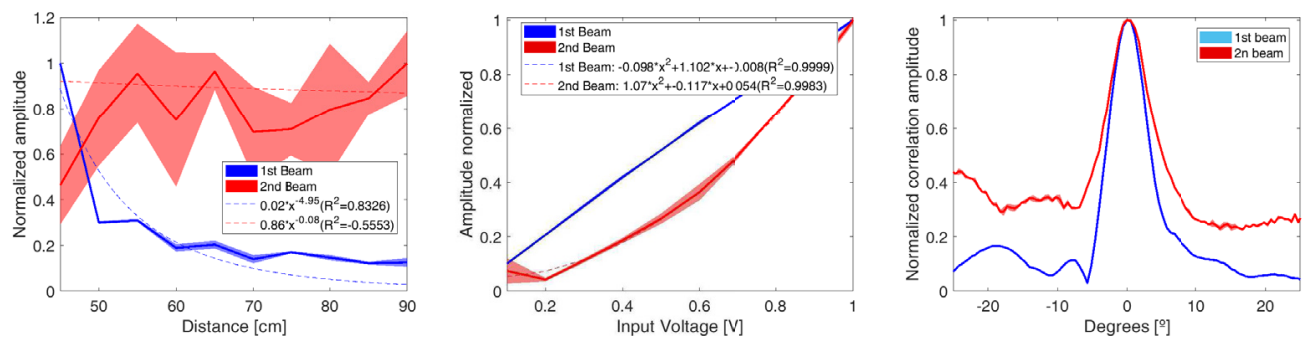

Figure 3. Attenuation (left), voltage variation (center) and directional (right) measures.

Moreover, the parametric technique has also been applied to directional acoustic communi-cations [6], and could also be implemented in neutrino telescopes.

\section{Conclusions}

Two applications of the parametric sources technique for neutrino telescopes have been described. In the first one, it has been shown that it is possible to mimic the acoustic signature of a UHE neutrino with a compact array validated with prototypes and tests. Simulations of an array with 5 elements present a directivity of $3^{\circ}$ Full Width Half Maximum $\left(\sigma=1.3^{\circ}\right)$. In the second one, it has been shown that the use of parametric emitters simplifies the facilities for the calibration of sensors, such as the acoustic sensor of the KM3NeT DOM that could be calibrated in water tanks of moderate size.

\section{References}

[1] Saldaña, M. PhD Thesis, Acoustic System Development for Neutrino Underwater Detectors. Gandia: Universitat Politècnica de València (2017)

[2] Ardid, M. et al. Sensors, Acoustic Transmitters for Underwater Neutrino Telescopes, 12(4) p4113-4132 (2012)

[3] G, Askaryan. Atomic Energy, Hydrodynamical emission of tacks of ionising particles in stable liquids, 3 p152 (1957)

[4] Saldaña, M. et al. Sensors, Transducer Development and Characterization for Underwater Acoustic Neutrino Detection Calibration 16(8) p1210 (2016)

[5] E.J.Buis; et al. Characterization of the KM3NeT hydrophone. ARENA2018 this issue.

[6] Campo-Valera, M; et al. Sensors, Acoustic Parametric Signal Generation for Underwater Communication 18(7) p2149 (2018) 